\title{
ROBERT FRANK
}

\section{La société française depuis 1945 Américanisation, européanisation, mondialisation et identité nationale}

Dans cette courte intervention, il s'agit, à propos des mutations bien connues de la société française depuis 1945, de suggérer quelques modestes réflexions sur les notions d'américanisation, d'européanisation, de mondialisation, et de poser la question de l'identité nationale française par rapport aux phénomènes que recouvrent ces notions polysémiques et riches en chausse-trapes. Souvent, on évoque, au sujet du malaise français depuis l'élection présidentielle de 2002 ou du vote négatif au référendum européen de 2005, une crise identitaire nationale: Est-ce à dire qu'elle est provoquée par ces transferts socioculturels venus de l'extérieur? Ou la notion de crise identitaire est-elle également ambiguë, au point de cacher plusieurs identités en crise, parmi lesquelles l'identité nationale n'est pas nécessairement la plus touchée?

\section{L'AMÉRICANISATION NE PRODUIT PAS AUTOMATIQUEMENT PLUS D'»AMÉRIQUE DANS LES TÊTES«1 (ANNÉES 1950 ET 1960)}

Quand on évoque l'américanisation, on pense d'abord à un mode de vie, à des pratiques socioculturelles. Une question importante se pose: les imaginaires sociaux sont-ils transformés - et comment? - par l'introduction de ces pratiques dans une société?

\section{ACCULTURATION DES MODES DE CONSOMMATION ET DES PRATIQUES SOCIOCULTURELLES VENUS D'AMÉRIQUE}

Par »américanisation «, on désigne le plus souvent le transfert de modèles de consommation, de modes de vie, de pratiques socioculturelles ou de cadres de pensée nés ou adoptés originellement aux États-Unis. Or, la principale importation d'Amérique qui transforme fondamentalement la société en Europe continentale et en France à partir des années 1950 et surtout à partir des années 1960 est certainement la consommation de masse. La Grande-Bretagne connaît ce début de changement dès les années 1930, ce qui lui permet d'être le premier pays européen à développer après la guerre, dès le début des années 1950, cette »société de consommation«. Celle-ci exprime bien dans

1 Denis LACORne, Jacques RUPNIK Marie-France TOINET (dir.), L'Amérique dans les têtes, Paris 1986. 
une large mesure une américanisation, puisque nombre des produits clés, des biens durables consommés en masse, ressortent de l'American way of life, ou s'identifient à lui, même si certains d'entre eux existaient sur le marché indépendamment des influences venues d'outre-Atlantique: l'automobile, le réfrigérateur - avec sa marque éponyme, le Frigidaire -, l'aspirateur, la radio, puis la machine à laver, la télévision. Ce ne sont pas seulement de nouveaux produits manufacturés, mais de nouveaux modes de vie induits, qui génèrent une révolution des mœurs, changent l'image et même la place de la femme dans la société, modifient l'espace familial autour du »living« (ou living room) tendant à remplacer dans les habitations françaises le couple »salon-salle à manger $\ll^{2}$.

De plus, avec la diffusion du pick up ou électrophone, des disques vinyle, des transistors, une culture de masse s'empare de la jeunesse, celle des teenagers. Le phénomène vient encore d'Amérique, avec le rock'n roll des années 1950. Le vecteur principal est la radio, avec l'émission »Salut les copains « diffusée par Europe n 1 à partir de 1959, relayée par le disque. Elvis Presley a ses émules en France, à travers un Johnny Hallyday dont le premier quarante-cinq tours sort en $1960^{3}$.

L'émergence des nouveaux modes de vie, ainsi que de cette nouvelle culture juvénile gagne toutes les couches de la société, y compris, avec des décalages, celles de la campagne. Il en résulte une relative homogénéisation sociale dans l'habillement, la mode, les styles de consommation et les pratiques culturelles. On a pu parler de »moyennisation « ou de »classe-moyennisation ${ }^{4}{ }^{4}$ de la société française, dans la mesure où une grande classe moyenne se substituerait progressivement à la bourgeoisie et à la classe ouvrière. La montée du secteur tertiaire (commerce, services, etc.), aux dépens du secteur secondaire (emplois dans l'industrie), celle des cols blancs aux dépens des cols bleus, commencée aux États-Unis, gagne l'Europe occidentale et la France. Cette tertiarisation est certes relative en France; elle n'empêche pas le secteur industriel de croître jusqu'en 1975 tant en valeur absolue qu'en proportion de la population active grâce à la forte baisse du secteur primaire ou agricole. Néanmoins, déjà prégnante dans les années 1960, elle devient dominante à partir de la deuxième moitié des années 1970 et contribue à ce phénomène de »classe-moyennisation«.

\section{Secteurs d'activités}

Secteur primaire

Secteur secondaire

Secteur tertiaire

\section{6}

$37 \%$

$29,5 \%$

$33,5 \%$
1954

$26,6 \%$

$35 \%$

$38,4 \%$

\section{0}

$13 \%$

$38,7 \%$

$48,3 \%$

\section{0}

$5,6 \%$

$28,9 \%$

$65,5 \%$
2001

$4,1 \%$

$22,7 \%$

$73,2 \%$

Cela dit, sans entrer dans des débats complexes, la notion de »moyennisation« est très discutée. L'évolution, on le sait, n'a pas été aussi simple. Les mutations, y compris celles qui sont induites par l'américanisation n'ont pas tué la »distinction sociale« en France qui, loin de disparaître, passe par d'autres chemins, changeant ses critères et ses

2 Dominique BoRnE, Histoire de la société française depuis 1945, Paris ${ }^{3} 2002$.

3 Jean-François SIRINELLI, Les baby boomers. Une génération 1945-1969, Paris 2003.

4 Henri MENDRAS, La France que je vois, Paris 2002. 
clivages selon des lignes moins visibles, mais réellement bien tranchées, malgré tout. Les antagonismes sociaux ont changé de nature, mais pas d'intensité. Si ce phénomène de «classe-moyennisation «, avec ses apparences ou ses réalités plus profondes, commence dans les années 1960, les antagonismes sociaux d'ancien type persistent et coexistent avec d'autres formes de lutte sociale: la contestation de mai 1968 mêle la lutte de classes traditionnelle, la grève massive, à d'autres formes de combats d'un type plus nouveau. D'ailleurs, la compréhension entre les acteurs du mouvement, ouvriers d'une part, et étudiants perçus comme bourgeois d'autre part, a été plus que difficile. Ce qui prouve que la »classe-moyennisation« à l'américaine est soit un concept non opératoire, soit un processus non achevé en France en 1968.

\section{AMÉRICANISATION À LA FRANÇAISE ET ANTIAMÉRICANISME EN FRANCE}

Le concept d'américanisation est d'autant plus difficile à cerner que bien des transformations économico-sociales et socioculturelles qui viennent d'être mentionnées procèdent au moins autant d'une vague de modernisation générale à l'Occident, et exportable hors de l'Occident, que d'une importation linéaire venue d'outre-Atlantique. Lorsque les GI's, dans le cadre de l'installation des bases de l'OTAN en France, découvrent au début des années 1950 qu'ils s'établissent dans un pays où l'habitat n'a pas toujours le »tout confort«, où les W.-C. sont encore trop souvent sales, collectifs, extérieurs au fond de la cour ou sur le palier, ils subissent un véritable choc culturel ${ }^{5}$. En 1954, 28\% des foyers seulement sont équipés de ces »commodités intérieures «, contre $73,8 \%$ en $1975 ; 5 \%$ des familles ont une douche ou une salle de bains en 1946 contre $70 \%$ trente ans plus tard. Voilà bien le principal trait, le plus visible sans doute de la »révolution invisible« française de ces »Trente Glorieuses«, si bien analysées par Jean Fourastié 6 . Cette mutation, si essentielle dans la vie quotidienne, comble le >retard ‘ du pays sur les États-Unis. Mais est-ce à dire qu'il s'agit là d'un effet de l'américanisation? Non, ce n'est pas le regard choqué du soldat américain qui a induit cette révolution sanitaire en France. La modernisation passe par des logiques qui ne viennent pas toutes d'Amérique.

Voilà en effet posée la question de la modernisation, de la convergence des modernités dans tout l'Occident, bref de l'occidentalisation tant à l'intérieur de l'Occident (sa relative homogénéisation dans la durée) qu'à l'extérieur (sa diffusion dans les autres régions du monde). Il s'agit là d'un processus complexe, non linéaire, multidirectionnel, qui implique des phénomènes de transfert, de circulation et d'appropriation. Dans cette dynamique de modernisation, les vents d'ouest sont certainement dominants, d'Amérique vers l'Europe, vers la France, du moins au XX $\mathrm{X}^{\mathrm{e}}$ siècle, et plus encore après 1945. Mais chaque société européenne se réapproprie à sa façon les novations, quitte à remettre en circulation, dans la direction inverse, le produit de son adaptation. D'autre

5 Jenny RAFLIK, Les décideurs français et l'alliance atlantique, thèse de doctorat sous la dir. de Robert Frank, université de Paris I Panthéon-Sorbonne, 2006.

6 Jean FouRASTIÉ, Les Trente Glorieuses ou la révolution invisible de 1946 à 1975, Paris 1979. 
part, la banalisation de pratiques venues d'ailleurs peut faire oublier cet »ailleurs«, et ce d'autant plus que la nouvelle pratique est enracinée, réappropriée, adaptée. L'automobile en masse à la française, c'est d'abord la $4 \mathrm{CV}$ Renault plutôt que la »belle américaine«, et la petite voiture à l'européenne peut à son tour concurrencer outre-Atlantique les véhicules de grande dimension. Johnny Hallyday, Eddy Mitchell et d'autres, inspirés de la musique venue des États-Unis dès la fin des années 1950, créent un rock bien français dans les années 1960 et 1970. Dans ce domaine, la circulation ne s'effectue pas dans le sens inverse, France-Amérique. C'est plutôt la révolution anglaise des Beatles et des Rolling Stones des années 1962-1966 qui permet le feedback Europe-Amérique par le truchement de la special relationship culturelle GrandeBretagne-États-Unis. Ce »moment britannique« transforme à son tour l'inspiration du rock américain, lui donnant une dimension plus sociale, plus contestataire, favorisant à son tour une musique pop qui prend en Amérique, à la faveur de la protestation contre la guerre du Vietnam, des aspects de contre-culture, la culture hippie dont le point d'orgue se situe au festival de Woodstock en 1969. La circulation continue, et l'influence touche encore l'Europe. En France, la phase du gentil yéyé de la première moitié des années 1960 cède la place à une musique plus contestataire ${ }^{7}$.

La réappropriation d'un modèle peut signifier certaines formes de fascination pour ce dernier, mais pas nécessairement une identification au pays qui l'a généré ni aux visions du monde qu'il tente d'exporter. La circulation entre pratiques culturelles et représentations n'est pas automatique. Les cadres de pensée sont forcément influencés par l'américanisation sociale, mais ne sont pas américanisés pour autant. L'américanisation de la société française atteint un de ses sommets pendant la période gaullienne, au moment même où se développe un certain antiaméricanisme politique et intellectuel 8. Américanisation et antiaméricanisme ne sont pas incompatibles, surtout en France. Du côté des contestataires français des »années 68 «, le paradoxe entre ces deux phénomènes n'est évidemment qu'apparent: leur antiaméricanisme est contre la politique de Washington et non contre la jeunesse américaine qui les influence; leur américanisation au niveau des pratiques culturelles devient précisément aussi politique, car leur critique radicale de l'»impérialisme américain« ne fait que rejoindre la remise en question exprimée par les étudiants américains eux-mêmes, dont les sit-in contre la guerre au Vietnam inspirent désormais certains rituels de manifestations en Europe et en France.

Néanmoins, par-delà les ambivalences de l'antiaméricanisme (admiration-rejet, fascination-répulsion), le discours antiaméricain reste en France plus virulent qu'ailleurs en Europe, ce qui ne veut pas dire que l'influence socioculturelle de l'Amérique soit moins forte dans ce pays. Au contraire, c'est sa vigueur qui peut susciter des phénomènes de rejet dans une partie de la société. Ce rejet renvoie, comme partout, à l'affrontement entre tradition et modernité (forcément étrangère). La question est de savoir pourquoi en France la tradition se construit sur des valeurs dites »nationales«

7 Jean-François SiRINELli (voir n. 3); Agnès TACHIN, La Grande-Bretagne dans l'imaginaire des Français. Opinions et représentations, 1958-1969, thèse de doctorat sous la dir. de Robert Frank, université de Paris I Panthéon-Sorbonne, 2004.

8 Philippe Roger, L'Ennemi américain: généalogie de l'antiaméricanisme français, Paris 2002. 
contre une modernité considérée comme venant de l'étranger, en l'occurrence de l'Amérique, pourquoi, dans le camp adverse, les modernistes se croient obligés d'invoquer le dieu d'outre-Atlantique - »Le Défi américain« en 1967 de Jean-Jacques Servan-Schreiber -, pourquoi le duel tradition-modernité, dont les Français n'ont pas le monopole, se cristallise chez eux en grande partie sur une guerre d'images FranceAmérique? Comme souvent, la représentation de l'»autre«, de l'altérité, s'explique davantage par la complexité et les conflits intérieurs de la fabrication de l'image de soi ${ }^{9}$. Les rapports entre américanisation et pro- ou antiaméricanisme dans une société sont à chercher dans les modes de construction de l'identité nationale.

Il serait bon de comparer ces phénomènes dans plusieurs États européens. Nul doute qu'en Grande-Bretagne les sentiments proaméricains dominent - bien qu'en France on sous-estime certaines formes de l'antiaméricanisme anglais - et qu'ils accompagnent l'américanisation de la société britannique d'autant mieux qu'ils valorisent l'identité nationale, affermie dans sa fierté d'avoir des liens culturels forts avec le cousin d'Amérique, avec qui, politiquement et militairement de surcroît, on a gagné la Seconde Guerre mondiale. Le poids de la mémoire de celle-ci compte en effet dans la construction de l'articulation complexe entre pratiques culturelles américanisées et représentations de l'Amérique dans les esprits des années 1950 et 1960. En Allemagne de l'Ouest, l'américanisation correspond à un fort désir d'occidentalisation après le drame de l'expérience nazie qui avait mis les Allemands hors du champ de l'Occident. L'américanisation est donc aussi vécue comme un chemin de la réintégration allemande dans la civilisation occidentale, besoin inutile chez les Britanniques et les Français. En revanche, ceux-ci, marqués par la défaite, par le syndrome de 1940, reconstruisent une identité nationale blessée sur des valeurs culinaires, gastronomiques, intellectuelles et politiques qui les distinguent mieux de l'Amérique avec laquelle ils vivent mal la relation de dépendance. La dialectique entre la forte américanisation de la société et la faiblesse de l'image de l'Amérique dans les têtes parait bien une spécificité française, du moins en Europe.

\section{L'EUROPÉANISATION DE LA SOCIÉTÉ FRANÇAISE TROUVE SON ÉQUILIBRE PENDANT LES TRENTE GLORIEUSES}

Dans la façon de se moderniser, les pays européens ont chacun une spécificité nationale, qui se retrouve dans la façon de se réapproprier l'américanisation. Mais il y a en même temps une convergence entre eux dans les modes d'appropriation que l'on peut appeler européanisation.

9 Robert FRANK, Introduction: Images et imaginaires dans les relations internationales depuis 1938, dans: Cahiers de 1'IHTP 28 (1994), p. 5-11; ID., Mentalitäten, Vorstellungen und internationale Beziehungen, dans: Wilfried LOTH, Jürgen OSTERHAMMEL (dir.), Internationale Geschichte. Themen - Ergebnisse - Aussichten, Munich 2000, p. 159-185. 


\section{LA NÉCESSITÉ DE DÉCOLONISER LE SENS ANCIEN ET COLONIAL DE L'EUROPÉANISATION}

Cette notion cependant pose problème par sa polysémie et elle ne signifie pas seulement convergence ou mise en convergence européenne. Il existe un premier sens qui date du temps des colonies et des empires: l'européanisation des populations que l'on appelait alors »indigènes «. S'habiller, manger, vivre »à l'européenne« était une forme d'européanisation hors d'Europe. Cette acception peut-elle avoir aujourd'hui en Europe une validité, appliquée à la question de l'intégration des immigrés? Même pour les derniers arrivés, la question fait débat: s'agit-il d'un problème d'intégration à une société nationale ou à une des sociétés européennes? Quant aux populations d'origine immigrée, installée depuis une génération, ou a fortiori davantage, ayant acquis la citoyenneté du pays, on ne peut parler d'intégration: la question est plutôt celle du »vivre ensemble« entre groupes, entre communautés, la question du racisme, du rejet social et des constructions identitaires ou contre-identitaires qui en découlent. Il serait incongru de parler d'européanisation - dans cette acception »coloniale« - comme solution à la révolte des banlieues en France parce que, précisément, c'est d'une »décolonisation« des concepts et des imaginaires dont la société française a besoin. Voilà sans doute d'ailleurs un des problèmes spécifiques français par rapport à l'Allemagne dans le traitement des relations intercommunautaires. Bref, cette ancienne signification n'est jamais enfouie très loin dans l'inconscient ou le subconscient collectif en France

\section{L'EUROPÉANISATION DES EUROPÉENS DANS LE CADRE DE LA CONSTRUCTION EUROPÉENNE}

Il est un autre sens plus récent du terme, en vogue surtout chez les politologues: l'européanisation d'aujourd'hui renvoie à ce qu'induit la Communauté européenne, puis l'Union européenne. Il s'agit de la communautarisation ou de l'»UEuropéanisation«, c'est-à-dire de l'impact des politiques communautaires, européennes sur chacun des États membres et sur leurs sociétés respectives. De là, trois angles différents. D'abord, quelles sont les convergences européennes suscitées par la construction de l'Europe, par la CEE, puis l'UE? Deuxièmement, plus largement - car l'impact des politiques européennes ne crée pas que des convergences -, quels changements en général sont générés dans les États membres par le développement de nouvelles lois, directives, normes et décisions? Comment se développe »le processus d'appropriation nationale de la contrainte européenne (c'est-à-dire les conséquences de la politique européenne sur la politique intérieure: dans les valeurs, les mentalités, les institutions, les procédures, les discours, etc.)« ${ }^{10}$ ? Enfin, en quoi la construction européenne européanise-t-elle les Européens, renforcent leur identité européenne, voire en crée une nouvelle? Nul doute que les politologues, comme Claudio Radaelli, ont raison de

10 Maxime LefEBVRE, Et si l'Europe se déconstruisait..., dans: Annuaire français des relations internationales 6 (2005), p. 234-248. 
mettre en perspective cette européanisation créée par l'UE elle-même, de refuser de réduire ce phénomène à la question des convergences ${ }^{11}$. Mais il est dommage que cette dernière problématique soit d'une part simplifiée par eux d'une façon linéaire - en fait, il convient de parler du couple indissociable »convergence-divergence« - et ne soit pas d'autre part suffisamment prise en compte par les politologues dans son épaisseur historique.

\section{L'EUROPÉANISATION DES EUROPÉENS AVANT LA CONSTRUCTION DE L'EUROPE}

L'européanisation intérieure de l'Europe ne commence pas en effet avec la construction européenne. Les travaux de Hartmut Kaelble montrent bien les critères de convergence sociale des pays européens au $\mathrm{XIX}^{\mathrm{e}}$ et au $\mathrm{XX}^{\mathrm{e}}$ siècle, avant l'aventure communautaire commencée le 9 mai 1950 et indépendamment de celle-ci, des critères qui ont fait émerger une »société européenne« originale par rapport à d'autres sociétés industrialisées, en particulier la société américaine: la structure familiale, le mariage tardif, le rapport à la ville et au centre-ville, la longue persistance d'une classe et d'une culture ouvrières, la force de l'État-providence, etc. ${ }^{12}$

\section{SOCIÉTÉ FRANÇAISE ET ÉQUILIBRE ENTRE EUROPÉANISATION, AMÉRICANISATION ET IDENTITÉ NATIONALE JUSQU'AUX ANNÉES 1970}

Il est certain que la société française partage toutes ces caractéristiques de 1945 jusqu'aux années 1970, et au-delà. La question est de savoir comment s'articulent les deux européanisations au deuxième et au troisième sens définis plus haut. D'abord, le long processus d'européanisation sociale au $\mathrm{XIX}^{\mathrm{e}}$ et au $\mathrm{XX}^{\mathrm{e}}$ siècle a-t-il favorisé le déclenchement de la construction européenne? À bien des égards, la réponse est affirmative. Mais, là encore, les automaticités sont à relativiser. Stanley Hoffmann a raison de constater que plus les convergences sociales européennes s'affirment, plus se confirment aussi les >idiosyncrasies« nationales, c'est-à-dire les modes nationaux d'appropriation de l'européanité ${ }^{13}$. Il en est de même avec l'adaptation et l'enracinement social de l'impact des politiques communautaires. Cela dit, celles-ci ont renforcé directement et indirectement nombre des spécificités sociales de l'Europe telles qu'elles ont été distinguées par Hartmut Kaelble. Pour la France, s'établit même dans les années 1960 un équilibre remarquable entre américanisation, européanisation

11 Claudio Radaelli, The Domestic Impact of European Union Public Policy: Notes on Concepts, Methods, and the Challenge of Empirical Research, dans: Politique européenne 5 (2001), p. 107-142; Vivien A. SCHMIDT, Europeanization and the mechanics of economic policy adjustment, dans: Christian LEQUESNE, Yves SUREL (dir.), L'intégration européenne. Entre émergence institutionnelle et recomposition de l'État, Paris 2004, p. 185-216.

12 Hartmut KaElBle, Vers une société européenne 1880-1980. Une histoire sociale, Paris 1990.

13 Stanley HoffmanN, The European Sisyphus. Essays on Europe 1964-1994, Oxford 1995. 
et identité nationale. Sous l'influence des États-Unis, la France ouvre ses frontières. Mais elle le fait modérément, dans le cadre d'un espace restreint, celui de la petite Europe. À la fois ouverte et protégée, elle fabrique sa société de consommation, inspirée de l'Amérique, tout en développant son système de sécurité sociale et son Étatprovidence, spécificité européenne. Celui-ci est élargi aux campagnes, elles-mêmes transformées et enrichies grâce à la PAC. Du coup, l'antiaméricanisme, lorsqu'il s'exprime, reste cantonné à la sphère politique, permettant une affirmation facile d'identité nationale qui ne remet pas fondamentalement en cause l'américanisation sociale ni l'européanisation économico-socio-politique. C'est cet équilibre qui est rompu à la fin du $\mathrm{XX}^{\mathrm{e}}$ siècle, sous le coup de la crise commencée dans les années 1970, mais qui ne fait sentir ses effets sur l'européanisation de la France que dans les années 1980 et 1990 .

\section{CRISE, MONDIALISATION ET ENJEUX DE L'EUROPÉANISATION DE LA SOCIÉTÉ FRANÇAISE (FIN XX ${ }^{\mathrm{e}}-$ DÉBUT XXI ${ }^{\mathrm{e}}$ SIÈCLE)}

\section{LES EFFETS DE LA CRISE}

Il n'est pas de notre propos d'analyser les causes de la crise économique qui débute en 1973-1974. Le principal effet sur la société est le chômage massif qu'elle engendre très vite: un million dès 1976, deux millions en 1982, puis trois millions en 1993. Cette situation de chômage durable a des conséquences délétères sur la société française. La désindustrialisation achève la »désouvriérisation« et la »moyennisation« de la France. Mais cette classe moyenne grandissante se diversifie de plus en plus dans la crise entre gagnants et perdants. Il convient de parler d'elle au pluriel et leur dérive contribue à la déstructuration du tissu social français ${ }^{14}$. Les recettes keynésiennes ne réussissent plus à faire revenir le plein emploi et l'État-providence est remis en question: il n'est plus considéré comme une solution, mais comme un problème. Les solutions néolibérales l'emportent. On en vient à parler d'un modèle anglo-saxon, puisque Margaret Thatcher au Royaume-Uni et Ronald Reagan aux États-Unis sont les premiers à les appliquer au début des années 1980, Certes, cette appellation, qui met les deux pays sous la même rubrique, paraît exagérée à bien des égards, car si le welfare state a été considérablement réduit en Grande-Bretagne, il conserve encore sa force ainsi que sa spécificité typiquement européenne par rapport à sa faiblesse en Amérique. Cela dit, sur le continent, l'autre modèle, le »modèle rhénan«, qui garde plus jalousement certaines formes de régulation par l'État ${ }^{15}$, connaît cependant une crise qui le remet en question. Bref, les spécificités de la »société européenne« (par rapport à la société américaine) analysées et repérées par Hartmut Kaelble dans les années 1980 paraissent décliner.

14 Louis CHAUVEL, Les classes moyennes à la dérive, Paris 2006.

15 Michel ALBERT, Capitalisme contre capitalisme, Paris 1991. 


\section{L'AMÉRICANISATION CÈDE LA PLACE À LA MONDIALISATION}

Cette »dés-européanisation« des Européens n'est que relative car elle ne profite pas nécessairement au modèle américain. Dans ce contexte qui contraste avec celui des Trente Glorieuses, le thème de l'américanisation a tendance en effet à décliner. Si la société de consommation continue de se développer en France, malgré la crise, avec de nouvelles générations de biens de consommation durables (du magnétoscope à l'équipement informatique), elle se banalise et le modèle n'apparait plus comme spécifiquement américain. La concurrence, les nouveaux modes de gestion entrepreneuriale, l'incitation à déréguler l'économie, les nouveaux produits ne paraissent plus venir exclusivement d'Amérique. C'est le thème de la mondialisation et de la globalisation qui prend le relais, et cette conscience de mondialisation se développe surtout après la chute de l'URSS.

\section{L'EUROPÉANISATION CHERCHE LA VOIE SPÉCIFIQUE D'UNE MONDIALISATION À VISAGE HUMAIN}

Face à la crise des années 1970 et 1980, les pays d'Europe occidentale réussissent à ne pas s'enfermer dans le repli national, comme ils l'ont fait pendant la crise des années 1930. Au contraire, la construction européenne se renforce.

La mondialisation à partir des années 1980 et surtout 1990 remet en cause le rapport société-État, c'est-à-dire une spécificité française ou une spécificité européenne. Les travaux du groupe de travail »Crises et conscience de crise depuis 1973« montrent toute une évolution intéressante en France et sur le continent ${ }^{16}$. Certains points sont connus. Il existe d'abord un fort décalage par rapport au Royaume-Uni de Margaret Thatcher. Alors que celle-ci prépare le pays à l'ouverture à la concurrence internationale et prend des mesures draconiennes, la gauche française, au pouvoir en 1981, relance au contraire l'économie par une forte hausse des salaires. François Mitterrand, on le sait, renonce à cette politique dès 1982, puis plus résolument en 1983. Dès lors, une nouvelle prise de conscience sociale se fait jour, y compris dans certains syndicats, comme la CFDT par exemple17: il n'est pas possible de lutter frontalement contre le chômage et de refuser tout plan de licenciement; la crise est intériorisée et la société commence, douloureusement certes, à comprendre que les mutations sont nécessaires et qu'elles passent par un redéploiement de la main-d'œuvre. L'émission télévisée »Vive la crise« en février 1984, animée par Yves Montand, et relayée par le journal de gauche »Libération«, vante même les vertus pédagogiques de la crise, présentée comme capable de libérer de nouvelles énergies. Il existe un exemple significatif de cette évolution. Dans le domaine de la sidérurgie, alors que le plan élaboré en 1977 par

${ }^{16}$ Les développements qui suivent sont inspirés du dossier »Crises et conscience de crise « publié par ce groupe de travail (Geneviève DREYFUS-ARMAND, Robert FRANK, Maryvonne LE PULOCH, Marie-Françoise LÉVY, Michelle ZANCARINI-FOURNEL) dans: Vingtième siècle. Revue d'histoire 84 (2004).

17 Ibid. Voir l'article de Frank GEORGI. 
le commissaire européen Étienne Davignon avait été rejeté en France par les salariés dans un climat de violence, sept ans plus tard, en 1984, le gouvernement nomme Jacques Chérèque, secrétaire général adjoint de la CFDT, comme préfet délégué chargé du redéploiement industriel18: la partie est loin d'être facile, mais la mutation s'effectue. La France ne va pas aussi loin que le Royaume-Uni, mais certains changements sont malgré tout acceptés. La contrepartie consiste à trouver une voie européenne à cette reconversion économique et sociale. Dans ce nouveau contexte, les Français attendent beaucoup de l'Europe, celle-ci devant servir de bouclier rassurant et d'interface efficace entre les vertiges de la mondialisation et l'enfermement du repli national. Toutes les réalisations européennes depuis 1984 vont dans ce sens, y compris la création de la monnaie unique, l'euro. Hartmut Kaelble montre comment, des années 1970 aux années 1990, l'identité européenne, au sens de sentiment d'appartenance à la Communauté ou à l'Union européenne, a progressé ${ }^{19}$. Créer plus de solidarité économique en Europe devrait permettre aux pays européens de mieux se protéger socialement contre la violence de la concurrence internationale. L'européanisation au niveau de l'UE ou »UEuropéanisation« a pour objectif de sauver les spécificités du modèle social européen et de donner une dimension humaine à la mondialisation.

\section{CONCLUSION \\ 2005: UNE CRISE EUROPÉENNE DE LA CONSCIENCE FRANÇAISE OU UNE CRISE FRANÇAISE DE LA CONSCIENCE EUROPÉENNE?}

Qu'en est-il aujourd'hui? L'américanisation fait moins problème aux Français que l'Amérique elle-même. Intégrée, banalisée, davantage de l'ordre de l'interaction et des transferts socioculturels, elle relève du mouvement plus général d'occidentalisation au plan culturel et de mondialisation au plan économique. Il existe bien quelques formes minoritaires de rejet dans des conjonctures bien spécifiques: la destruction d'un Mc Donald's à Millau en 1999. Cet acte n'empêche pas cependant la fréquentation française croissante de cette chaîne à restauration rapide. Et les divergences des Français avec la politique extérieure de George W. Bush ne ralentissent pas ce mouvement d'acculturation; d'ailleurs, ces tensions suscitent moins d'antiaméricanisme politique en France que de francophobie aux États-Unis.

C'est le rapport de la société française à l'européanisation qui fait problème depuis le rejet du projet de traité constitutionnel européen par 55\% des électeurs en mai 2005. Les novations institutionnelles n'ont pas vraiment fait l'objet de débat, moins que la partie III qui ne faisait que réunir les dispositions déjà appliquées en matière de marché et de politiques économiques confirmant une Europe libérale déjà existante. Le refus de celle-ci par une grande partie de la gauche qui, jusqu'alors, approuvait la construction européenne, a été la cause déterminante de la victoire du »non« et il est significatif d'une crise à multiples facettes. D'abord, la persistance de ce chômage depuis plus de

18 Ibid. Voir les articles de Xavier VIGNA et de Françoise BERGER.

19 Ibid. Voir l'article de Hartmut KAELBLE. 
trente ans, qui détruit la société de l'intérieur, crée de la précarité, déstabilise les familles, les banlieues, sans que des solutions apparaissent, suscite une grande désespérance. Même l'Europe, comme horizon d'attente, déçoit, puisque les espoirs des années 1980 et 1990 de la voir réguler la mondialisation se sont évanouis. Le bouclier de l'UE qui devait protéger les sociétés européennes ne semble pas fonctionner. Pour beaucoup, ce qui avait été accepté vingt-deux ans plus tôt - difficilement d'ailleurs -, à savoir le sacrifice par François Mitterrand de la »politique économique de gauche« sur l'autel de l'Europe, est désormais rejeté. Le »retour du refoulé« de 1983 a joué à plein en 2005. La campagne référendaire a été dominée, dans cette gauche déçue de l'européisme, par le débat sur l'Europe sociale. Aux yeux de beaucoup, celle-ci reste à construire et elle leur paraît désormais la condition préalable à toute autre avancée dans l'intégration européenne.

Il reste à identifier la nature profonde de ce rejet de 2005. S'agit-il d'un repli identitaire des Français sur la France? À bien des égards oui, mais cela ne signifie pas nécessairement une crise d'identité nationale. Celle-ci ne se porte pas si mal, même si la révolte des banlieues de novembre 2005 parait la mettre en question. En fait, cette révolte révèle davantage un besoin de reconnaissance d'intégration à la nation qu'un rejet de celle-ci. C'est la société française qui est en crise, non point la nation, les multiples identités sociales des citoyens, plus que l'identité nationale. L'inquiétude suscitée par la mondialisation, et maintenant par la représentation d'une Europe ultralibérale, ne va pas jusqu'à pousser la majorité des Français à repousser l'UE. Ce n'est pas le fait d'être européen en France qui pose problème, mais la façon de l'être, et les débats sur la manière divisent les Français. L'identité européenne de la grande majorité d'entre eux n'est pas remise en question, mais ils s'interrogent sur leur rapport à cette identité. La conscience européenne des Français reste intacte et l'européanisation a imprégné la société, mais la régulation française de cette acculturation européenne est en difficulté à cause du mal-être des identités sociales. Bref, il y a moins crise européenne de la conscience française que crise française de la conscience européenne.

\section{DEUTSCHE ZUSAMMENFASSUNG}

Amerikanisierung ist ein kulturelles Phänomen, das sich auf gesellschaftlicher Ebene durchgesetzt hat: Der massive Transfer soziokultureller Praktiken und die Einführung amerikanischer Produkte - Fernsehen, Kühlschrank, Auto oder Waschmaschine - haben die französische Gesellschaft seit den 1950er und 1960er Jahren stark verändert. Allerdings bleibt die Frage, ob dieser Wandel nun als spezifische Amerikanisierung oder als generelle Modernisierung oder Verwestlichung zu bezeichnen ist bzw. in welcher Beziehung die verschiedenen Phänomene zueinander stehen. Auf jeden Fall hat es sich nicht um einen linearen, sondern um einen komplexen, mehrseitigen Prozeß gehandelt, der nationalspezifische Variationen der Akkulturation, des Transfers und der Wechselbeziehung aufweist. Zudem ist die Amerikanisierung in der französischen Gesellschaft stets ambivalent betrachtet worden: Einerseits öffnete sich das Land vor allem aus wirtschaftlichen Gründen - nicht zuletzt in der Ära de Gaulle -, andererseits empfand man dies als latente oder sogar manifeste Bedrohung der eigenen Identität - gerade in den Jahren 1958 bis 1969. Bis heute ist die Dialektik zwischen starker Amerikanisierung der Gesellschaft und gleichzeitiger mentaler Distanz zu den USA ein französisches Spezifikum, zumindest in Europa. Des- 
halb stellte die Europäisierung bereits seit dem 19. Jahrhundert, ergänzt durch die »EUisierung« nach 1950, mit der Folge einer Europäisierung der Amerikanisierung, auch immer ein wichtiges Zwischenglied zwischen nationaler und transnationaler Ausrichtung dar - eine Funktion, die allerdings mittlerweile verloren gegangen ist: Das Ende der »Trente Glorieuses« im Zuge der neuen Weltwirtschaftskrise seit Mitte der 1970er Jahre bedeutete auch das Ende des klassischen und spezifischen europäischen Sozialstaats, allerdings nicht zugunsten einer weiteren Amerikanisierung; vielmehr sind die aktuellen Reaktionen auf die Krise, wie wachsende Deregulierung, wirtschaftsliberale Konkurrenz, neue Unternehmensstile, eher Zeichen einer umfassenderen Globalisierung. Gleichwohl herrschte gerade in Frankreich noch lange Zeit die Hoffnung auf einen europäischen Weg aus der Krise mit Hilfe einer wirtschaftspolitischen Europäisierung oder $»$ EUisierung«. Das französische »Non« zum EU-Verfassungsvertrag vom Mai 2005 belegt, daß eine Mehrzahl der Franzosen sich in dieser Hoffnung getrogen sieht. Gleichwohl stehen in Frankreich weder die europäische Identität noch die bisher bereits eingetretene Europäisierung grundsätzlich zur Disposition, wohl aber das »Wie« der europäischen Akkulturation angesichts der durch die Globalisierung bedingten neuen weltwirtschaftlichen Herausforderungen. 